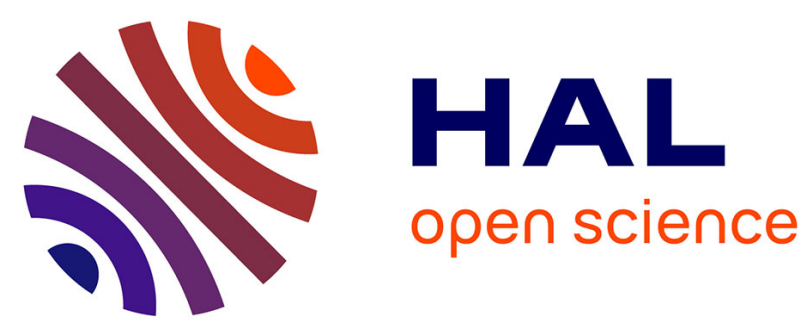

\title{
Identification of six open reading frames in the Salmonella enterica subsp. enterica ser. Typhi viaB locus involved in $\mathrm{Vi}$ antigen production
}

\author{
Hervé Waxin, Isabelle Payant, Suzan Kolyva, Michel y Popoff
}

\section{To cite this version:}

Hervé Waxin, Isabelle Payant, Suzan Kolyva, Michel y Popoff. Identification of six open reading frames in the Salmonella enterica subsp. enterica ser. Typhi viaB locus involved in Vi antigen production. Research in Microbiology, 1993, 144 (5), pp.363-371. 10.1016/0923-2508(93)90193-6 . hal-01600906

\section{HAL Id: hal-01600906 https://hal.science/hal-01600906}

Submitted on 1 Jun 2020

HAL is a multi-disciplinary open access archive for the deposit and dissemination of scientific research documents, whether they are published or not. The documents may come from teaching and research institutions in France or abroad, or from public or private research centers.
L'archive ouverte pluridisciplinaire HAL, est destinée au dépôt et à la diffusion de documents scientifiques de niveau recherche, publiés ou non, émanant des établissements d'enseignement et de recherche français ou étrangers, des laboratoires publics ou privés. 


\title{
Identification of six open reading frames in the Salmonella enterica subsp. enterica ser. Typhi viaB locus involved in $\mathrm{Vi}$ antigen production
}

\author{
H. Waxin, I. Virlogeux, S. Kolyva and M.Y. Popoff ${ }^{(*)}$ \\ Unité des Entérobactéries, Unité INSERM 199, Institut Pasteur, 75724 Paris Cedex 15
}

The Vi antigen of Salmonella enterica subsp. enterica ser. Typhi (hereafter referred to as Typhi) is a capsular polysaccharide consisting of a homopolymer of $\alpha$-1,4 2-deoxy-2N-acetyl galacturonic acid (Felix and Pitt, 1934). Determinants of $\mathrm{Vi}$ antigen occupy two widely separated chromosomal loci, designated viaA and viaB. The viaB locus, specific to $\mathrm{Vi}$ expressing strains, maps at $92 \mathrm{~min}$ on the chromcsome of Typhi (Johnson et al., 1965). Cloning and molecular analysis of this chromosomal region were reported previously (Hashimoto et al., 1991; Kolyva et al., 1992). Here we report on the nucleotide sequence of a 9.3-kb fragment located within the Typhi strain Ty2viaB locus.

The dideoxy chain-termination method, using modified T7 DNA polymerase ("Sequenase" ; USB Corp.) and universal forward and synthetic primers, was employed after subcloning of appropriate restriction fragments into M13 derivatives (Messing and Vieira, 1982). All

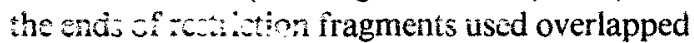
one another. DNA sequencing was performed at least twice on both strands. Nucleotide sequence data were analysed using the LipmanPearson program (Lipman and Pearson, 1985). The sequence data have been submitted to the EMBL data library and were assigned accession no. X67785.
As shown in figure 1, examination of the DNA sequence revealed six open reading frames (ORF), termed: tviA (Typhi $V i)$, tviB, tviC, $t v i D, t v i E$ and $t v i F$. All were transcribed in the same crientation. Their description is reported in table I. Significant homology was detected between TviB protein and AlgD (Deietic et al., 1987), a GDP-mannose dehydrogenase of Pseudomonas aeruginosa (26\% identity and $71 \%$ similarity in 288 amino acids overlap) and between TviC protein and StrE (Pis:owotzki et al., 1991), a TDP-glucose dehydratase of Streptomyces griseus ( $28 \%$ identity and $68 \%$ similarity in 326 amino acids overlap). A lipoprotein signal sequence (Hussain et al., 1982) with a potential cleavage site for signal peptidase II (see fig. 1) was found in the TviF sequence. TviF protein shared significant homology to BexD (Kroll et al., 1990) and CtrA (Frosch et al., 1991) proteins $(27 \%$ identity and $72 \%$ similarity in 280 amino acids overlap) which are involved in capsule export by Haemophilus influenzae and Neisseria meningiaidis, respectively.

Studies designed to determine whether TviB and TviC proteins are involved in the biosynthesis, and TviF in the biogenesis, of the Vi antigen are presently under way in our laboratory.

Key-words: Vi antigen, UKF, Subinontuta ser. Typhi; viaB locus, Nucleotides, Sequencing; Note.

Submitted May 7, 1993, accepted Mäy i4, is93.

(*) Corresponding author. 
Table I. Description of the six open reading frames identified within the 9.3-kb sequenced segment of the viaB locus.

\begin{tabular}{ccccc} 
Open reading frames & \multicolumn{2}{c}{ Location (nucleotide) } & \multicolumn{2}{c}{ Expected protein } \\
from: & to: & a & mm \\
\hline tviA & 770 & 1306 & 179 & 21099 \\
tviB & 1554 & 2828 & 425 & 47612 \\
$t$ viC & 2834 & 3877 & 348 & 39478 \\
$t$ viD & 4285 & 6396 & 704 & 80402 \\
$t$ viE & 6399 & 8132 & 578 & 64902 \\
$t$ viF & 8180 & 9244 & 355 & 38877 \\
\hline
\end{tabular}

$\mathrm{aa}=$ number of amino acids; $\mathrm{nm}=$ predicted molecular mass in dalton.

\section{Identification de six phases ouvertes de lecture dans le locus viaB impliqué dans la production de l'antigène Vi chez Salmonella enterica subsp. enterica ser. Typhi}

L'antigène Vi de Salmonella enterica subsp. enterica ser. Typhi est un polyoside capsulaire dont l'expression est contrôlée par les loci chromosomiques $v i a A$ et $v i a B$. La séquence d'un fragment $(9,3 \mathrm{~kb})$ du locus via $B$, qui est spécifique des souches produisant l'antigène Vi, a été déterminée. Six phases cuvertes de lecture $(t v i A, t v i B, t v i C, t v i D, t v i E$ et $t(i F)$ codant respectivement pour des polypeptides de 21.099, $47.612,39.478,80.402,64.902$ et 38.877 daltons ont été mises en évidence. Sur la base des résuitats de comparaison de séquence, les protéines TviB, TviC et TviF pourraient être impliquées dans la biosynthèse et la biogénèse de l'antigène Vi.

Mots-clés: Antigène $\mathrm{Vi}$, ORF, Salmonella ser. Typhi; Locus viaB, Nucléotides, Séquençage; Note.

\section{References}

Deretic, V., Gul, I.F. \& Chakrabarty, A.M. (1987), Pseudomonas aeruginose infections in cystic fibrosis: nucleotide sequence and transcriptional regulation of the algD gene. Nucl. Actds Res.. 15, 4567-4581.
Felix, A. \& Pitt, R.M. (1934), A new antigen of B. typhosis. Lancet, 227, 186-191.

Frost 1. M., Edwards, U., Krausse, B. \& Weisgerber, C. (1. 991$)$, Evidence for a common molecular origin of the capsule gene loci in Gram-negative bacteria expressing group Il capsu'ar polysaccharide. Mol. Micribiol., 5, 1251-1263.

Hashimoto, Y., Ezaki, T., Li, N. \& Yamamoto, H. (1991), Molecular cloning of the viaB region of Salmonella typhi. FEMS Microbiol. Letters, 90, 53-56.

Hussain, M., Ichihara, S. \& Mizushima, S. (1982), Mechanism of signal peptide cleavage in the biosynthesis of the major lipoprotein of the Escherichia coli outer membrane. J. Biol. Chem., 257, 5177-5182.

Johnson, E.M., Krauskopf, B. \& Baron, L.S. (1965), Genetic mapping of $\mathrm{Vi}$ and somatic antigenic determinants in Salmonella. J. Bact., 90, 302-308.

Kolyva, S., Waxin, H. \& Popoff, M.Y. (1992), The Vi antigen of Salmoneila typh:: molecular analysis of the via $B$ locus. I. gen. Microbiol., 138, 297-304.

Kroll, J.S., Loynds, B., Brophy, L.N. \& Moxon, E.R. (1990). The bex locus in encapsulated Haemsphilus influenzae: a chromosomal region involved in capsule polysaccharide export. Mol. Microbiol., 4, $1853-1862$.

Lipman, D.J. \& Pearson, W.R. (1985), Rapid and sensitive protein similarity searches. Science, 227 , 1435-1441.

Messing, J. \& Vieira, J. (1982), A new pair of M13 vectors for selecting either DNA strand of double-digest restriction fragments. Gene, 19, 269-276.

Pissowotzki, K., Mansouri, K. \& Piepersberg, W. (1991), Genetics of streptomycin nrodurtion in Streptrmyer griseus: molecular structure and putative function of genes strELMB2N. Mol. gen. Genet., 231, 113-123.

Fig. 1. Nucleotide sequence of a $9.3 \mathrm{~kb}$ fragment of the Salmonella ser. Typhi Ty2viaB locus.

The putative Shine-Dalgarno sequences ate underlined. The cleavage site of the lipoprotein signal peptide at amino acid position 17 of TriF is indicated with a star. These sequence data were assigned accession no. $X 67785$ in the EMBL data library. 
GAA TTC TGC AAA CCA GCC CTG TAC CAT CAA GTT CAT ATC CTC AGC GGA GGA AAT GCG TGA

$\infty$

AAT CCA GCG CAA CAC ACG GAG TAT CAC OGA CTA CCO AGG CAT CAT GAT TCI CAG ARA CCT

150

180

CAA ATG CTT CGC CGG CAT GAA TAA GGT ATT CAT TTE CGA AGC AGT CAC GCA CCA TCA TTT $210 \quad 240$

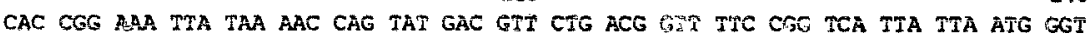
270

GAC TCT AAT GCA AAC ATC TAG CGA GAA AAT ATT TTG TAG ACA TCG TCA ACG GAA AAG TTA $330 \quad 360$

ACT AAC AAT AGT CAT TAT CCÀ TAT CTT TAA TTT GAA ATG GAA ACA GGG CAA AGA GTA ATT 390

420

AAA ATA CAG CCT GTG ATT CTG TAC AGT TAP GG TTA ACC CCA AAG GTG CAA ACA ATT AAA $450 \quad 480$

TTT GTA ACA GAT TAT TTC AAA TAC GAT TAG GAA TAT TCT TAT TTT GAG GAA TGG CTT AGC 510

ATT ITT TAT GET CGC CAG GAT TAA AAA ATA CGC CCT ATC CAA ACA ATT TAG CAT AAT TTA 570

TCA TTC GAT TTT CTA GAC TAA ATZ AGA TTT TTT GAT AGG TAC AAA CAA TGA ATT GTG CAG 630

GTT TGA CAT CAA TTC TCT GTT GTG TAA AAA TCC CGT ITA GGC CGT TAG TAC TAT TAA AAT $690 \quad 720$ TAG GGT AAT AAT TTT ATT GTT AGT TAA TTG TTA ACA GGA GCA AAG AAT TAG ATA TTG CTT $750 \quad$ TVIA 781

GTA CCT TTT CTA TGG ATG AAA TTA GGT TAT TTC AGC hTA AGGAG ACTTC ATG AGG TTT CAT Met Arg Phe His

811

841

CAT ITC TGG CCT CCG AAT GAT ATC TAT TTC GGG GTT GGA GCT GCT GGC ATT ATT GAA GAA

His phe Trp Pro Pro Asn Asp Ile Tyr Phe Gly Val Gly Ala Ala Gly Ile Ile Glu Glu 872

901

GTG TCA CTG ATA ACA AAT GAC AGA AAT TAT TTG TTT GTG AAC CTA AAT̃ CGC TAC AGC CTG Val Ser leu Ile Thr Asn Asp Arg Asn Tyr Leu pho val Asn Leu Asn Arg Tyr ser Leu 931

TTA AAT GCC CTG AAT TTT TTC ACG CGA ATG AGT GAT ATT AAT AAA ATA ATC GTT ATC ATT Leu Asn Ala Leu Asn phe Phe Thr Arg Met Ser Asp Ile Asn Lys Ile Ile Val Ile Ile c91 1021

TCA AGT TCG CGA CTA ATG CCC CTT GCA CGT TTT TGG TTG ACA GAG TGC AAA AAT GTT ATT Ser Ser Ser Arg Leu Met Pro Leu Ala Arg the Trp Leu Thr Glu Cys Lys Asn Val Ile 1051

1081

GCT GTT TTC GAT GCG GCA ACA TCA GTC CAG GAT ATT ATC AGA AAT GTC AGT CAA CAC CAA Ala Val phe Asp Ala Ala Thr Ser Val Gin Asp Ile Ile Arg Asn Val Ser Gln His Gln 1111

1141

AGT GGT GAA AAG ATC TTG ACG GAG CAG AGA GAT TAT CGT TTC AGA ATT AAC CGT AAG GAT Ser Gly Glu Lys Ile Leu Thr Glu Gln Arg Asp Tys Arg Phe Arg Ile Asn Arg Lys Asp 1171

1201

ATA GTA AAG ATG AAA TAT TTC CTT TCG GAA AGT GGT ATE GAA GAG OT'S CAG GAT AGA TT'T Ile Val Lys Met Lys Tyr phe Leu Ser Glu Ser Gly Met Glu Glu Leu Gin Asp Arg Phe 1231

ATG AAC TCA TCA TCG ACT ATG TAT CGC TGG AGA AAA GAA TTG GCA GTA AAA TTX GGA GTA Met Asn Ger Sez Ser The Met Tyr Arg Trp Arg Lys Glu Leu Ala Val Lys phe Gly val 129121320

CGT GAG CCG CGC TAT CTG TTA TTG CCG GAT TCA GTT ACT TTA CTG TAA TGC GGT AAT TT hrg Glu Pro Arg Jyr Leu Leu Leu Pro Asp Ser Val Thr Leu Leu 1350

TTA TTG AGT AAA ACA CGG ACA AGT ATT TCG TTT CAG CAC MAA ATT ATT TSC GTT ACT CAT 1410

TGG CGT TAA TAC ATA TAT TCT CAG CGA CTT CTG TTC IAT TCA AGT AAG AAA GGG GTA CGG $1470 \quad 1500$

TTA TAC GTT TTC ATT AAC CAT ACT GGC TGC TAC GGC CAG GGG CGG TAG CGT ATC TGA ATA $1530 \quad$ tVIB 1562 AAC ACC TAG AAT TAA CTT TGT AAA TAT AAA ATT TIA GTA ABGGA TTAA TAAGA GTG TTC GGT

ATA GAC GAG GTh AAA ATC GCG ATT ATT GGG CTG GGA TAT GTT GGG CTT CCT CTG GCA GTT Ile Asp Glu Val Lys Ile Ala Ile Ile Gly Leu Gly Tyr Val Gly Leu Pro Leu Ala Val 
GAA TTT GGC AAA TCT CGT CAG GTT GTT GGC ITC GAC GTT AAT AAA AAG TGT ATT CTT GAA Glu Phe Gly Lys Sef Arg Gin Val. Val Gly phe Asp Val Asn Lys Iys Arg Ile Leu Glu 1712

TTA AAG AAT GGG GTG GAT GTC AAT CTG GAA ACC ACT GAA TAR GAA TTA CGT GAG GCT

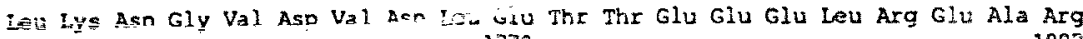
1772

IAT CTG AAA TTT ACT TCC GAG ATT GAG AAG ATC AAA GAA TGT AAT TTT TAC ATC ATC ACC Tyr Leu Lys phe Thr ser Glu Ile Glu lys Ile Lys Glu Cys Asn Phe Tyr Tle Ile Thr 1832

1862

GTC CCG ACG CCG ATA AAT ACC TAC AGG CAA CCA GAC CTC ACC CCA CTA ATC AAG GCC AGT Val Pro Thr pro Ile Asn Thr Tyr Lys Gin Pro Asp leu Thr Pro Leu Ile Lys Ala Ser 1892

1922

GAA ACC GTT GGT ACA GTG CTG AAT CGG GGA GAT ATT GTG GTA TAT GAA TCT ACG GTA TAT Glu Thr val Gly thr Val Leu Asn Arg Gly Asp Ile val Val tyr Giu Ser thr Val Tyr 19521982 CCG GGA TGT ACC GAA GAA GAA TGC GTG CCG ATC CTT GCT CGT ATG TCC GOA ATG ACT TTC Pro Gly Cys Thr Glu Glu Glu Cys Val Pro Ile Leu Ala Arg Met Ser Gly Met Thr Phe 2012

2042 AAC CAG GAT TTC TAT GTC GG'T TAT AGC CCG GAA AGG ATC AAT CCC GGT GAT AAA AAG CAC Asn Gln Asp the Tyr Val Gly Tyr Ser Pro Glu Arg Ile Asn Pro Gly Asp Lys Lys His 2072

2102

CGT TTA ACC AAC ATC AAG AAA ATC ACC TCC GGT TCA ACC GCA CAG ATC GCC GAA Arg Leu Thr Asn Ile Lys Lys Ile Thr Ser Gly Ser Thr Ala Gin Ile Ala Glu Leu Ile 2132

2162

GAT GAA GTA TAT CAG CAG ATC ATC AGC GCA GGT ACA TAT AAA GCA GAG AGC ATC AAA GTT Asp Glu val Tyr Gln Gin Ile Ile Ser Ala Gly Thr Tyr Lys Ala Glu Ser Ile Lys Val 2192 2222 GCT GAG GCA GCG AAG GTG ATT GAA AAT ACG CAA CGC GAT CTG AAT ATT GCC TTG GTC AAT Ala Glu Ala Ala lys Val Ile Glu Asn Thr Gln Arg Asp Leu Asn lle Ala leu val Asn 2252 2282

GAG CTG GCG ATT ATT TTT AAT CGT TTA AAT AIC GAT ACT GAA GCC GTG CTA CGT GCC GCT Glu Leu Ala Ile Ile phe Asn Arg Leu Asn Ile Asp The Glu Ala Val Leu Arg Ala Ala 2312 2342 GGC AGE AAA TGG AAT TTC CTG CCA TTC CGT CCG GGA CTG GTC GGT GGT CAC TGT ATT GGC Gly Ser Lys trp Asn phe Leu Pro Phe Arg Pro Gly Leu Val Gly Gly His Cys Ile Gly 2372 2402 GTA GAT CCC TAT TAT CTG ACA CAT AAA TCT CAG GGC ATT GGC TAT TAT CCA GAA ATC ATA val Asp poo Tyr Tyr leu The His bys Ser Gln Gly lle Gly Tyr Tyr pro Glu Ile Ile 2432 2462 CTT GCA GGA CGC CGC CTG AAC GAC AAC ATG GGC AMC TAT GTC TCC GAG CAG TIG RTC AAA Leu Ala Gly Arg Arg Leu Asn Asp Asn Met Gly Asn Tyr Val ser Glu Gin leu Ile Lys 2492 2522 GCA ATG ATC AAA AAA GGA ATT AAC GTT GAG GGT TCC AGC GTG CTG ATT CTC GGC TTT ACC Ala Met Ile Lys Lys Gly Ile Asn val Glu Gly Ser Ser val Leu Ile Leu Gly Phe Thr 2552

2582

TTT AAA GAA AAC TG' CCG GAC ATC AGA AAT ACA CGC ATT ATT GAT GTG GTA AAG GAA CTC Phe Lys Glu Asn Cys pro Asp Ile Arg Asn Thr Arg Ile Ile Asp Vai val lys glu Leu 2612

2642

GGT AAA TAT AGT TGT AAA GTG GAT ATT TTT GAT CCA TGG GTG GAT GCC GAA GAG GTA AGA Giy lys Tyr ser Cys lys Val Asp Ile phe Asp pro Trp val Asp Ala Glu Glu Val Arg 2672

2732

CGA GAG TAT GGC ATT ATC CCG GTA TCG GAA GTC AAA TCA AGC CAC TAC GAT GCG ATC ATT Arg Glu Tyr Gly Ile Ile gro Val Ser Glu Val Lys Ser ser His Tyr Asp Ala Ile Ile 2732 2762 GTT GCA GTA GGA CAT CAC CAA TII AAN CAU ATG GGN NOT CNG GAT AT2 CGC GEO TTC GGA Val Ala Val Gly His Gin Gln the Lys Gin wet Gly Ser Glu Asp Ile Atg Gly Phe Gly 2792 2822

AAA GAT AAA CAT GTA CTT TAT GAT TTG AAG TAT GTT CTT CCG GCT GAG CAG TCA GAT GTG Lys Asp Lys His Val Leu Tyr Asp Leu lys Iyr Val Leu Pro Ala Glu Gln Ser Asp Val tric 2851 2881

AGA TTG TAA TC ATG ACG GCI TAC GAA GAA CTA CGG ACC AAA GTG GIT CTG GCA CCA AAG Arg LeU Met Thr Ala Tye Glu Glu Leu Arg Thr Lys leu Val Leu Ala Pro Iys 2911 2941 CGC TGG CTG ATC AC' 2 GGC GTA GCA GGC TTT ATT GGC TCC GGC TTA TTA GAA GAA TTA CTC Arg Trp Leu Ile Thr Gly Val Ala Gly phe Ile Gly Ser Gly Leu Leu Glu Glu Leu Leu 
TTT CTC ARO 3001 Phe Leu Asn Gin me Val Ile Gly leu Asp Asn Phe Ser Thr Gly Tyr Gin kis Asn Leu 3031

उ०ธิ

GAC GAC GTT CGC ACO TCC GTC AGT GAG GAG CAA TGG TCG CGA TTT ATT TTT ATT CAG GGT Asp Asp Val Arg Th: Ser Val Ser Giu Glu Gln Txp ser arg the the phe Ile Gin Gly 3091

$3 \geq 21$

GAC ATC AGG AAA TIT ACT GAC TGT CAG AAA GCG TGT AAG AAC GTT GAC TAT GTT CTC CKC Asp Iie Arg Lys phe Thr Asp Cys Gin Lys Ala Cys Lys Asn Val Asp Tyr Val Leu his 3151

3181

CAA GCC GCG CTA C: HEC GTG CCA CGT TCC CTA AAG GAT CCC ATC GCG ACT AAT AGC GES Gln Ala Ala leu $6:$, Val Pro Arg Ser leu Lys Asp Pro Ile Ala Tht Asn Ser Ala 3211 3241

AAT ATT GAT GGT TT" Th AAT ATG TTG ACG GCG GCG AGA GAT GCT CAT GTC TCT AGT TTC Asn Ile Asp Gly Phe heu Asn Met Leu Thr Ala Ala Arg Asp Ala His Val ser Ser phe 3271 330 :

ACC TAC GCC GCA AGC AGT ACE ACC TAT GGA GAC CAT CCC GAT TTA COT AAA ATT GAG GAA Thr Tyr Ala Ala Ser Ser Ser thr Tyr Gly Asp His Pro Asp zeu Pro Lys Ile Glu Glu 3331

336.

CGG ATC GGT CGA CCA CTC AGC CCG TAT GCG GTA ACA AAA TAC GTC AAT GAA TTG TAC GCT Arg Ile Gly Arg pro 3391

3421

GAT GTG TTT GCA CGT TAT GAA TTT AAC GCT AAT GGC CTA CGC TAC TIT AAT GTC TTT Asp val Phe Ala Arg: Tyr Glu phe Asn Ala Ile Gly Leu Arg Tyr the Asn val phe 3451

3481

GGT CGC CGC CAA

Gly Arg Arg Gln

Asn Gly Ala Tyr Ser Ala Val Tle pro Arg Trp Ile Leu Ser 3511

3541

CTT CTT MAA GAT GAA CCN ATT TAT ATC AAT GGC GAT GGC TCA ACA AGC AGG GAT TTT TGC Leu Leu Lys Asp Glu $F: 3$ Tle Tyr Ile Asn Gly Asp Gly Ser Thr ser Arg Asp Phe Cys 3571

3601

TAT ATA GAG AT: GTG Tyr Ile Glu Ast: Val 1 le in Ala Asn leu leu Ser Ala the Thr Asn Asp leu Ala ser 3531

3662

AAA AAT AAG GF TAT AA: GTG GCA GTT GGA GAT AGA ACT TCG ITA AAT GAG CTT TAT TAT bys Asn Lys Val Tys Ash Val Ala Val Gly Asp Arg the ser Lew Asn Glu Leu ryo Tyt 3691 3721

CTA ATI CGL GAT GGG CTT AAT TTA TGG CGG AAC GAA CAA AGT AGA GCT GAA CCA ATT TAT Leu Tle Arg Asp bly Leu Asn Leu Trp Arg Asn Glu Gln Ser Arg Ala Glu Pro Ile Ty" 3751

3781

AAA GAT TTT CGT GAC CE: \$AC GTT AAG CAT AGC CAG GCA GAT ATT ACC AAA ATA AAA ACA Lys Asp ohe Arg Asp Gly tsp Val Lys his ser Gln Ala Asp Ile Thr Lys Ile Lys Tht 3812 3841

TTT CTT TCA TAT GAG CCT GAA TTT GAT ATC AAA GAA GGA CTT AAG CAG ACT CTA AAA TGG Phe leu ser Jyr Gin Pr. Clu the Asp Ile Lys Glu Gly Leu Lys Gin Thy Leu Lys Irp 3871 3901

TAT ATC GAT MAA CAT $\therefore$ TTE TAT TCC TCG CTA TAA CTA CTC ACT TTC CTT TCA CGM Tyr Ile Asp Lys His Ser hr led Tyr Ser Ser $y_{z}$ ? 3931 3961

GGA TGA ATT TAA TGA DAT JTE CAG GGA TGT TTA COC TTA CAG CCA TTG GCA GTT GCC GTA 3991 THA COO TTA CAO CCA

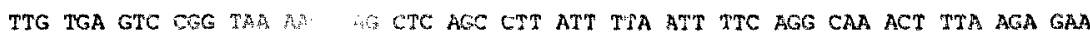
4051

4081

TAT ATE GTT TIA TS

WA GCA GGG AGG CAT TAC AGC AGA TTC GGT ITA TTT TGG GCT 4111

TGA TAG ACA FTC WTO : WGE TCC CAC CAT TIA TIT ITA GAC CTA ATG TAA ACT ATT CAA $4171 \quad 4201$

ACA CCG ACG TAC AT 4231

4261

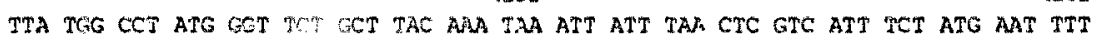
tV $1 D 4290$

4320

IT AGC CAA AC ACAG COE TOS TEC ITG TAC TGG TCG TTG GCC ACG CAA GGA AAT CGA CAC

Met hyr Trp Ser Leu Mla The Gin Gly Asn Arg His 4350

4380

AAA CTU CTE GCS TA2 CTE AAA GAC GAT CCC TGT TTT GCC GGA ATG TCG GAA GAC GAT CGT Lys Leu Leu Ala Tyz Le' tys Asp Asp Pro Cys phe Ala Gly Met Ber Glu Asp Asp Arg 
4410

4440

GCC TTA TTA AGC Aat atC AAT GTC GAG CAG ATG GAT GAG CAT GCT ATC GAA CAG GAT ATG Ala Leu Leu Sez Asn Ile Asn Val Glu Gln Met Asp Glu His Ala rle Glu Gln Asp Met 4470

4500

ATG GAA ATC GTT CAG CTT CTT GGT CGC GAT CGC GTT ATG TTT ATG ACA CAT GTT GAT GCC

Net Glu Ile Val Gin Leu Leu Gly Arg Asp Arg Val Met phe Met Thr his Val Asp Ala 4530

GTG ACT CGT GCT GGA ACC GTC ATT CTA TCC CGT AGT CGG TTG ATT AAA AAT GTC GAC ACC Val Thr Arg Ala Gly Thr Val Ile Leu Ser Arg Ser Arg Leu Ile Lys Asn Val $\mathrm{Asp}$ Thr 4590

4620

ATC GCC GCC AGG ATG GAT ATT CCC TGC GTT AAC CCG ACA AAT TTG ATG GAA AAG TGG GGG Ile Ala Ala Arg Met Asp Ile fro Cys Val Asn Pro Thr Asn Leu Met Glu Lys Trp Gly 4650

4680

CAG AAA CGA GCC CTS GAA AAA AAT GGC GAC GAT CTT ACT CAT TAT ACC GAT ATG TTT GGT Gln iys Arg Ala Leu Glu lys Asn Gly Asp Asp Leu Thr His Tyr Thr Asp Met phe Gly 4710 4740 GAC GCG ATC GTT GCG GCT ATT TTT AAG GGA GTG ATC AAT AAT ACT AAT CAT CAT CTT GAT Asp Ala Ile Val Ala Ala Ile Phe Lys Gly Val Ile Asn Asn Thr Asn His His Leu Asp 4770 4800 GAG GGE CGA CAA GAG AAA CAG GAC CAA ATC AGT GAG ATT ACC TTA TCG ATC ACT AAG CAG Glu Gly Arg Gin Glu hys Gln Asp Gin Ile Ser Glu Ile Thr Leu Ser. Ile Thr Lys Gin $4830 \quad 4860$

CTT GCA GAT GGC GAC ATT ATT GCT GCA TCA CAA CAA CTT TTT GCC GCA TTA AGA AAT CAG Leu Ala Asp Gly Asp Ile Ile Ala Ala Ser Gln Gln Leu Phe Ala Ala Leu Arg Asn Gln 4890 4920

CAG CAA GAT CCC GTT CTA ATC CAA CTT GGG TCC GTA ATC TTC AGC CAT TTA GGT TAT TAT Gln Gln Asp pro Val Leu Ile Gin Leu arg ser val Ile phe Ser His leu Gly Tyr Tyr 4950 4980

GAA CAG GCT TAT CAG GAT ATT AGT GAT GTT GAG AAA ATT ATC GGT ACG ACT GAC AGT ACA Glu Gln Ala Tyr Gin Asp Ile Ser Asp Val Glu lys Ile Ile Gly thr The Asp ser Thr S010 5040

TTA CGT TGT CGG CTG AGG TCT CTA CAT GGA TTA GCG CGT TGG CGG GAA GCC TTA TCG ACG Leu Arg Cys Arg Leu Arg Ser Leu His Gly leu Ala Arg Trp Arg Glu Ala Leu Ser thr 5070

5100

GCA GAS ATG ATG CTT TCC AAT GAA ATT GAA GAT GAA GAA GTC CTT ACC GTỸ GCC GCC GGC Ala Glu Met Met Leu Ser Asn Glu ile Glu Asp Glu Glu val Leu Thr Val Ala Ala Gly $\$ 130$

5160

TCA GCC GAT GCT TTA CAG CTG TTT GAT AAG TCA TAT CAT TAT TGG AAA CGT GTA CTA TTA Ser Ala Asp Ala Leu GIn Leu phe Asp Lys Sex Tyr His Tyr Trp Lys Arg Val Leu leu 5190 5220

TTG AAT CCT GAA ACT CAA AGC GGA TGGS GTT AAT ITC CTG AGC AGC ACG Leu Asn Fro Glu Thr Gln Ser Gly Trp Val Asn Phe Leu Ser Ser Thr Gln Tyr Phe Asn 5250

5280

GAT GGC AAC GCA TIE ICT GAA GCT TTC CAT GCC GGC ATT CAA TCG CAG CGC CTA AAT GAT Asp Gly Asn Ala phe Ser. Glu Ala phe His Ala Gly Ile Gin Ser Gin Arg Leu Asn Asp 5310 5340 ACG TTT ATG GAA ACG GCG TTA TCT TTG GCA ATC AAA TTC AGT GAT GAA TTG ATT TTC ATG Thr phe Met Glu thr Ala Leu Ser Leu Ala Ile lys phe Ser Asp Glu Leu Ile Phe Met 5370

5400

CAT GCG CTC GAG CAG CTA CTC CGC CAT GAG TCA GAA TTT GCG CTG ACG GTA ITG TCG ACG His Ala Leu Glu Gin Leu Leu Arg his Glu ser Glu Phe Ala Leu Thr Vad Leu Ser Thr 5430 $\$ 460$

ATT CAT GAT ACC GGT CTC GTT ATC CGC ACA GCT TTC TGC ATC MG MAT ATG hCC TAT CAT Ile his Asp Thr Gly Leu Val Ile Arg Thr Ala Phe Cys the Lys Asn Met ser Iyr His 5490 5520

CAA GCG CTI CGC ACC TCG TAT AAA GAT AAA ATC CAC GAC GTT TTT GAG GCA TGG AAC AAT Gin Ala leu Arg thr Ser Tyr Lys Asp lys Ile His Asp Val Phe Glu Ala Trp Asn Asn 5550 5580

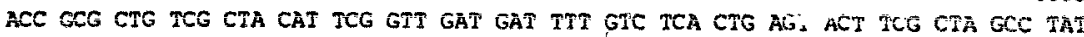
Thr Ala Leu Ser Leu His Ser Val Asp Asp phe Val Ser Leu Ser Thr Ser Leu Ala Tyr 5610 5640

AGC TAC TCT GCA TIT ATG GTT TAT CCC CAT TCA CGT ATP TCT CGC TTT AAT AAT GAA GTT Ser Tyr Ser Ala phe Met Val Tyr Pro His Ser Arg Ile Ser Arg phe Asn Asn Glu Val 5670 5700

AAA ATG GCA TGG CGC GAT AAA ITA AGA GAA ATG TAT GAG CGT GAG GAT TAT GAA AAT ATC Lys Met Ala Trp Arg Asp Lys Leu Arg Glu Met Tyr Glu Arg Glu Asp Tyr Glu Asn Ile 
CTG GCA GGG GCG AAA ATA GTG TGG CCA CIT CTG AAG ITT GAI CCC GTT GGC ACC GTA TAT Leu Ala Gly Ala Lys Ile val Trp pro leu Leu Lys phe Asp Pro val Gly thr Val Tyr 5790

TGT GCA AGA ACG GTG GTG AAT CTT GGT GCC TGG AAA GAC GCG TGC ACG TTG GCC CAC ATG Cys Ala Arg Thr Leu Val Asn Leu Gly Ala Trp Lys Asp Ala Cys Thr Leu Ala Hís Met 5850

$5 \$ 80$

ACC TTG ATT CGT AAC TCG AAC ATT ACC AGC CTG CAG TCG ATT ATG TTA CGC AGC ATA CGT Thr Leu Ile Arg Asn Ser Asn Ile Thr Ser Leu Gln Ser Ile Met Leu Arg Ser Ile Arg 5910

5940

CAT ATT AAC AAC ATT CCG TTC CTC ATT GAT TTG ATT GCT AAC GTC ATG AGC ATT ACT CTA His Ile Asn Asn Ile Pro Phe Leu Ile Asp Leu Ile Ala Asn val Met Ser Ile The teu 5970

6000 TCA TTC CAG AAT GCC ICA ATG AAC AAG TTG TIT GAG AAA GAG TGT CGC AAT GTT GCA ACC Ser phe Gln Asn Ala Ser Met Asn Lys Leu phe Glu Lys Glu Cys Arg Asn Val Ala thr 6030

6060

AGA GCC CTT AAA TAT GTA CGC CAG AAG AAA ACT GAA GGG CGT CTG GAT GAA GCA ITG TCT Arg Ala Leu lys Tyr Val Arg Gin lys Lys Thr Glu Gly arg Leu Asp Giu Ala Leu Ser 6090

6120

GTA TTG ATT AGC CTG AAA CGA ATT GAG CCT GAT GTT TCT CGT CTG ATG CGT GAA TAT AAG Val Leu Ile Ser Leu Lys Arg Ile Glu Pro Asp Val Ser Arg Leu Met Arg Glu Iyr Lys 6150

CAA ATT ATC AGA TTA TTT AAT GAG TCA CGE AAG GAT GGC GGT AGC ACT ATC ACG TCT TAT Gln Iie Ile Arg Leu Phe Asn Glu Ser Arg Lys Asp Gly Gly Ser Thr Ile Thr Ser Tyx 6210

6240

GAA CAT CTA GAC TAT GCG AAA AAA TTA CTC GTT TTT GAT AGC GAA AAT GCC TAT GCC TTG Giu His Leu Asp Tyr Ala Lys Lys Leu Leu Val Phe Asp Ser Glu Asn ala Tyr Ala Leu 6270 6300

AAA TAT ICC GCA TTA AAT ICA ATI; CAT TTA CGC GAC TAC ACG CAG GCT TTS CAG TAT TGG Lys Tyr Ala Ala Leu Asn Ala Met His Leu Arg Asp Tyr Thr Gln Ala Leu Gin Tyr Trp 6330 6360

CAG CGA CTG GAG AAA GIG AMT GSA CCA ACG GAG CCG GTG ACA AGG CAG ATC TCG ACG TGC Gin Arg leu Glu lys Val Asn Gly Pro Thr Glu Pro Val Thr Arg Gln Ile Ser Thr Cys 6390 tvis 6419

ATA ACC GCA TTA CAA AAA AAT ACA TCA GGG AAG TCG TA ATG ATT ACG CAG GAA GAA AAG Ile thr Ala Lel Gln wys Asn Thr ser Gly Lys Ser Met tle Thr Gin olu Glu Lys 6449 6479 TTA GCT GCA CTA GGA AAA ACG TGT TTA ACA TTA AMA CAA GAG AAG AAG CTT GCG CAA GCT Leu Ala Ala Leu Gly hys Thr Cys Leu Thr Lau Lyb Gin Gli lys i,ys Leu Ala Gln Ala 6509 6539 GTT GCG TTA ATT GAC AGT GAA TTA CCG ACT GAG GCI TTA ACT TCA TTA GCG ATG CTA AAA Val Ala leu Ile Asp Ser Glu Leu Rro The Glu Ala Leu Thr Ser leu Ala Met Leu Lys 6569

6,599

AAA GCA GAG TTY CTT CAT GAT GTC AAT GAA ACG GAG CET GCA TAC GCG CTC TAC GAA ACG Lys Ala Glu Phe Leu His Asp Val Asn Glu Thr Glu Arg Ala Tyr Ala lru Tyr Glu Thr 6629

6659

CTG ATT GCA CAA AAC AAT GAT GAA GCA CGT TAT GAG TAT GCA CGT CGT TTA TAT AAT ACG Leu Ile Ala Gin Asn Asn Asp Glu Ala Arg Tyr Glu Tyr Ala Arg Arg bat Tỵ Asn Thr 6689

6319

GGG CTA GCC AAA GAT GCT CAG CTA ATT CTT MAA ANG GTT ACE AAT GET GTG GAG AAA AAA Gly Leu Ala Lys Asp Ala Gin Lew Ile Leu Lys hys Val Ser Asn Gly Val Gln Lys Lys 6749 6779 TAT AAC AAT TAT TTA GGC AAA ATA AAT AAG ATC TOT OAT TTG CTT GAA CGC CTT GAA GGG Tyr Asn Asn Tyr Leu Gly Lys Ile Asn lys Ile Cys Asp Leu Leu Glu Ary Lay Glu aly 6809 6839 DAA GCG ATC CCY GTG GGG ACC AAC ACC TGT ATT ATT GCA ATG AKG CAT GC ATC TTE TTC

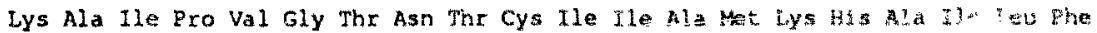
6869 6899

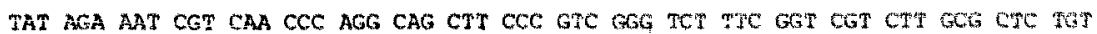
Tyr Arg Asn Arg Gin Pro Arg Gin heu pro Val Gly ser the Gly arg hel Ala leu cys 6929 6999

ACT GGC TCG CTA GGT ASC GGT GOT GCA GAG CGT CAG ATT TCE ASG CTE GWT ATC GH MTE

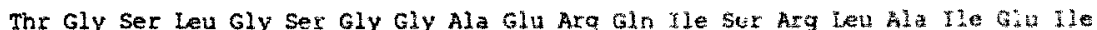
6989 7019 GCC AGA AAA TAT CGG CAA AAG $6 G G$ AAA ATT GGC GGC CTG AAT GA GA GA CCG

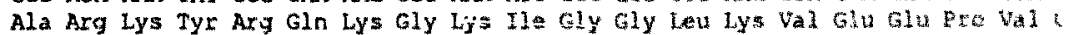


CTA ATT ATT CGC ICC CTG ACA CCG GAA CTC AGG CAA GAC TIT TTC CTG AAA GAA GTG CTG Leu Ile Ile Arg ser Leu Thr Pro Glu leu Arg Gln Asp phe Phe Leu Lys Glu Val Leu 7109

GAA GAA CAG GTC GAG GTT CTI GAA ATC GCG AAG ATT ACC GGA AAC TIG TIT GAC GAT GCG Glu Glu Gin Val Glu Val Leu Glu Ile Ala Lys Ile Thr Giy Asn Leu Phe Asp Asp Ala 71697199

ACA ATA GAA TCT CCA GAG TTG CGC TTA TTG CTA TCG CAT CTA CCG CCG GTG TGT AAA TAC Thr Ile Glu Ser Pro Glu leu Arg leu Leu Leu Ser his Lell Pro pro Val Cys Lys tyr 7229

7259

GGC ATC AAG CAT CTG GTC CCC CAT TTA TGC GAG CGC AAG CTG GAT TAT CTC TCC GTT TGG Gly Ile Lys His leu Val Pro His Leu Cys Glu Arg lys leu Asp Tyr leu Ser Val Trp 7285

7319

CAG GAT GGC GCT TGT CTG ATG ATT GCG CTI GCA GCA TTG ATT GCT GGC GTG CCC AGA ATT Gin Asp Gly Ala Cys Leu Met Ile Ala Leu Ala Ala Leu Ile Ala Gly Val pro Arg Ile 7349

7379

CAA CTG GGA TTA CGT GGG TTA CCG CCG GTG GTT AGA AAG CGT UTG TTC AAG CCG GAA TAT Gin Leu Gly Leu Arg Gly Leu Pro Pro Val Val Arg lys Arg Leu Phe Lys pro Glu Tyr 7409

GAG CCT CTC TAC CAG GCG CTG GCG GTC GTG CCT GGC GTT GAT TTT ATG AGT AAC AAC CAT Glu Pro Leu Tyr Gln Ala Leu Ala Val val Rro Gly Val Asp Phe Met Ser Asn Asn His 7479

TGT GTE ACT CGC CAT TAT GCC GAC TGG CTG AAG TTG GAG GCG AAC GAC ITC CAG GTT GTA Cys Val thr Arg His Tyr Ala Asp Trp Leu Lys Leu Glu Ala Asn Asp phe Gin Val Val 75297559

TAT AAC GGC GTC TTA CCG CCA TCT ACT GAA CCC TCT TCT GAG GTG CCA CAT AAA ATC TGG Tyr Asn Gly Val Leu Pro pro Ser The Glu Pro Ser ser Glu Val pro his lys Ile Trp 7589

7619

CAG CAG TTT ACG CAA AAA ACC CAG GAT GCG GAC ACG ACT ATT GGT GGC GTT TTC CGC TTT Gin Gin Phe Thr Gin Lys Thr Gin Asp Ala Asp Thx Thr Ile Gly Gly Val phe Arg Phe 7649

7679

CTA GGC GAT AAG AAC CCI TIT GCA TGG ATT GAT ITT GCA GCA COC TAT TTA CAA CAC CAC Val Gly Asp Lys Asn Pro phe Ala Trp Ile Asp Phe Ala Ala Axg Tyr Leu Gin His His 7709

7739

CCC GCC ACG CGC TTT GTG CTG GTA GCC GAT GGT GAT TJPA CGC GCT GAA GCG CAG MAA CGC Pro Ala Thr Arg phe Val leu Val Gly Asp Gly Asp Leu Arg Ala Glu Ala Gin Lys Arg 7759

7799

GCC GAA CAG TTA GGG ATT CTG GAG AGA ATA CTA TTC GTT GGC GCC TCG CGT GAC GTA GGG Ala Glu Gln Leu Gly Ile Leu Glu Arg lle Leu Phe Val Gly Ala Ser Arg Asp Val Gly 7829

7859

TAT TGG CTG CAA AAA ATG AAT GTA TTC ATT TTG TTT TCG CGT TAT GAA GGG CTA CCT AAT Tyr Trp Leu Gin Lys Met Asn Val phe Ile leu phe Ser Arg Tyr Glu Gly Leu Ero Asn 7889

7919

GTG CTT ATT GAA GCA CAA ATG GTC GGG GTG CCG GTG ATT TCA ACC CCT CCA GGT GGA TCG Val Leu Ile Glu Ala Gly Met Val Gly Val pro Val Ile Ser Thr pro Ala gly Gly Ser 7949 7979

GCA GAA TGC TTT ATT GAG GGT GTT TCG GGT TTC ATT CTT GAT GAT GCA CAG ACG GTG AAT Ala Glu Cys phe Ile Glu Gly Val Ser Gly phe Ile Leu Asp Asp Ala Gln Thr Val Asn 8009

8039

CTT GAC CAG GCT TGC CGC TAT GCA GAA AAG TTG GTC AAT TTA TGG CGC AGC AGA ACC GGT Leu Asp Gln Ala Cys Arg Tyr Ala Glu Lys Leu Val Asn Leu Trp Arg Ser Arg Thr Gly 8069 8099

ATT TGC CAA CAG ACG CAG TCA TTT TTA CAA GAA CGC TTC ACC GTG GAA CAT ATG GTG GGA Ile cys Gin Gin thr Gln Ser Ehe leu Gin Glu Arg the thr Val Glu His Met val Gly 8129

ACG TTT GTA AAA ACC ATT GCC TCT CAG CCT CGT TAA TTA ATG GGC ATC ATT TTT CAG CTAT Thr the Val Lys Thr Ile Ala Ser Gin Pro Arg

tVIF 8191

TTCA TTT ATA AAA TAA GTT ATG AAA AAA ATC ATC ATA TTA CTA ACG ACA TTT TTC CTG CTT Met Lys Lys Ile Ile Ile Leu leu Thr Thr Phe phe Leu teu 8251 B281

TCG GGA TGC ACT ATT CCC AGG GCG GTA TTT AAA TCC AGC CTT ATT AAT CAG GAC GAT CCT Ser Gly Cys The Ile pro Ang Ala Val Phe Lys Ser Ser Leu Ile Asn Gln Asp Asp pro 8311 8341

CGT TAT AAT CTG GTC GAA GTC ACG CCG ACA TTA AAA CTA AGC GCT CCC GAT ACT GTG CCG Arg Tyr Asn Leu Val Glu Val Thr Pro Thr leu lys Leu Ser Ala Pro Asp Thr Val Pro 
AAA ACT ATT GTC GAT CCG GTT TTT GCC GCA AAT AAC TGG CAC TGG ACA TCT TTG GCT AAA Lys Thr Ile val Asp Pro Val phe Ala Ala Asn Asn Trp his Trp Thr Ser Leu Ala Lys 8431

B461

GGC GAT GTG CTG CAT ATC ACT ATI TTA TCC JCG GGC GGG GCT GGA TAT TTA TCC AAT AAC Gly Asp Val Leu tis Ile thr Ile Leu Ser Ser Gly Gly Ala Gly Tyr Leu Ser Asn Asn 8491

8521

GCG AGC GGC GAC CGT GCG GAT TTT GAA AAT ATT CTI GTG ACT GAC AGT AAT ACC GTT CAG Ala Ser Giy hop Arg Ala Asp Phe Giu Ásn Ile Leu Val Thr Asp Ser Asn Thr Val Gln 8551

8581

GTG CCT TAT GCC GGG ACA ATC CCG GTT KCT GGA TTG GAT GTG ACG CAA CTG GCT GAT GAG Val Pro Tyr Ala Gly Thr Ile Pro Val Ser Gly Leu Asp Val Thr Gin Leu Ala Asp Glu 8611 8642

ATC AAA AAG CGA CTT TCG CGC GTT GTC CTG AAT CCT CAG GTG ATT GTG ACA CTT ACC GCC Ile Lys bys Arg Leu ser Arg Val Val Leu Asn Pro GIn Val Ile Val Thr Leu Thr Ala 86718701

CGC ACC GGA GCC ATG GTG ACG GTC GAG GGC AGC GGG AAA ACG GGT CGA TAC CCT CTC GAA Arg Thr Gly Ala Met Val Thr Val Giu Gly Ser Gly bys thr Gly Arg Tyr Pro Leu Glu 8731 8761

CAG AGT ATG AAT CGT CTG AGT CAT CTT TTG GCA ACG GCA GTG GCA GTA GAA AIT ACC AGC

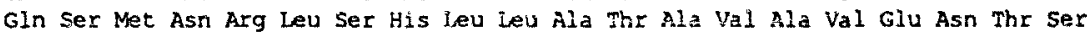
8791

8821 ACA GAT ATG ATG GAA GTT CAC GTG ACG CGC CAG CAG CAT TAT TTC ACT GCG CGA CTG ICA Thr Asp Met. Met Glu Val His val Thr Arg Gin Gln His Tyr Phe Thr Ala Arg Leu Ser 8851

8881

GAT ATT TAT CAG TAT CCC GGA TTG GAT ATI GCC TTA CAG CCG GAT GAC CGT ATC ACT CTG Asp Ile Tyr Gin Tyr Pro Gly Leu Asp Ile Ala Leu Gin Pro Asp Asp Arg Ile thr Leu 8911

3941

CGT CAN GTG ACC GAG TAT GTT AAT GTG CTC GGC GCA GCA GGT GTT CAG GGG AAA CAT GCT Arg Gln Val Thr Giu Tyr Val Asn Val Leu Gly Ala Ala Gly Val Gln Gly Lys His Ala 8971 9001 CTG GTT CAG CGT CAT TCC AGC GTC GTG GAT GCT TTG GCG CTC GCG AAA GGA ITA AAT GAC

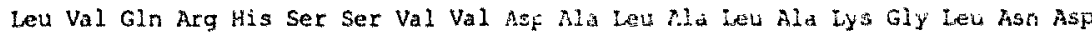
9031 9061

AAT CTT GCC GAT CCG CAA GCG ATT TTT CTG TAC AAC CAT AAC GAA GCG GAA CAG GCG AAA Asn Leu Ala Asp pro Gln Ala Ile phe Lel Tyr Lys His Asn Glu Ala Glu Gln Ala Lys 9091 $\$ 121$

CAG CAG ATG CGT AAG CTG MAT ATC TAT CAT GTC GAT ATG AGC CAA CCT AAC TCG GTG TTT Gln Gin Met Arg Lys Leu Asn Ile Tyr his Val Asp Met Ser Gin Pro Asn Sex Val phe 9151.9181

TTA GCG CAG GCC ATA CGA GTG GAC AAC GGC GAT GTT ATC TAT ATC TCG AAC GCE TCT TTG Leu Ala Gln Ala Ile Arg Val Asp Asn Gly Asp Val Ile Tyr Ile Ser Asn Ala Ser Leu 9211 9241

ACT GAT TTC GCI AAG GTG AAA GCC GCA TTC GAT AGC TTT TTG ACC CGC GGC ACT AAT TCT Thr Asp phe Ala Lys Val Lys Ala Ala Phe Asp Ser phe Leu Thr Arg Gly Thr Asn Ser 9271

9301

TIC TAA TGT CGC CAT ATG AAT ATT TTG AAA AAT AAT TCT TAT TAT TTI ATG AAA CTC ATT Phe 